\title{
Structural Analysis of The Receptor Binding Motif of Spike Glycoprotein Explains The High Stability Of SARS- CoV-2 RBD/hACE2-complex
}

\author{
Akshay N Pandya ${ }^{1}$
}

\section{Abstract}

SARS-CoV and SARS-CoV-2 both are

the Coronaviruses causing Severe Acute Respiratory Syndrome(SARS). Genomes of both SARS-viruses are identical and they both use Spike glycoprotein to enter the host cell. hACE2(human Angiotensin-Converting Enzyme) is the receptor used by both viruses to enter the host cell. Despite the similar structures, the SARS-CoV-2 is more infective compared to the SARS-CoV. As the SARS-CoV-2 $\mathrm{RBD} / \mathrm{hACE} 2$ complex is more stable compared to the SARS-CoV RBD/hACE2 complex, the SARS-CoV-2 is more effective in terms of entering the host cells. Structural analysis revealed that the addition of a Furin cleavage site and the structural changes in Receptor Binding Domain(RBD) of the Spike protein are mainly responsible for this increase in the stability. Differences between the structure of the Spike glycoproteins of SARS-CoV and SARSCoV-2 are discussed in-depth and an attempt to correlate them with increased infectivity of the SARS-CoV-2(compared to the SARS-CoV) is made.

\section{Keywords}

- SARS-CoV

- SARS-CoV-2

- Spike protein

- hACE2(human Angiotensin Converting Enzyme 2)

- Furin

- TMPRSS2(Transmembrane protease, serine 2)

\section{Introduction}

$\mathbf{E}$ irst Coronavirus identified was

Infectious Bronchitis Virus(IBV). It was discovered in 1932(1). Although the first Coronavirus was identified in 1932, Coronaviridae was proposed as a taxonomic family 30 years later after human Coronaviruses were identified in human patients with $\operatorname{cold}^{(2)}$.

Coronaviruses are enveloped viruses that contain the largest linear genome of known positive-sense single-stranded viral RNA(2). They are named for the crown-like spikes(Spike glycoproteins) on their surface. SARS-CoV and SARS-CoV-2 both are classified under the $\beta$ Coronavirus subdivision, Coronavirinae subfamily, Coronaviridae family, and Nidovirales order(3-5). Nidovirales earned this name as the viruses belonging to Nidovirales order have the capacity to produce a 3'multiplexed complex of subgenomic messenger RNA(mRNA), "Nidus" is a Latin word, which means "To nest"(2).

The Coronaviridae family infects more than 200 different hosts. Most terrestrial and marine animals and humans, including dolphins, birds, cattle, fish, and many more are affected by these viruses ${ }^{(2)}$. The Coronaviruses infecting mammals appeared about 7000-8000 years ago(6). In most cases, the infection caused by these viruses is considered as severe infection(2). In humans, these viruses cause respiratory tract infections which range from mild to lethal. Mild illnesses include some cases of the common cold, while more lethal varieties can cause SARS, MERS, and COVID-19(7). 
There are seven Coronavirus species having the ability to infect humans. Four common viruses, $\mathrm{HCoV}-229 \mathrm{E}$ and $\mathrm{HCoV}-\mathrm{NL} 63$ belonging to $\alpha$-Coronaviruses, $\mathrm{HCoV}-\mathrm{OC} 43$ and CoV-HKU1 belonging to the $\beta$-Coronaviruses, cause mild infections. The other three highly pathogenic viruses, SARS-CoV, MERS-CoV, and SARS-CoV-2 belong to the $\beta$-Coronaviruses ${ }^{(4,8-}$ 10). According to an epidemiological study, about $15 \%$ of common colds are caused by Coronaviruses in adults(11).

SARS-Coronaviruses are spherical shaped particles of about $125 \mathrm{~nm}$ diameter(12). In November 2002, the first cases of atypical pneumonia caused by the SARS-CoV appeared in Guangdong Province of China(13). During that SARS pandemic, 8422 people from 32 different countries were infected and 919 of them died(11\%)(14). The end of the pandemic was declared in Beijing as the last batch of 18 patients was recovered and discharged on June $20,2003^{(13,15)}$.

On December 29, 2019, A cluster of cases of atypical pneumonia caused by an unknown pathogen(later identified as SARS-CoV-2) was reported to health authorities of Hubei Province, Wuhan, China(16). A total of 70,91,659 people are found infected by this virus mainly due to person to person spread and 4,06,195 deaths are reported till now ${ }^{(17) .}$

The main route of transmission is respiratory droplets, but according to a study that demonstrated the presence of the virus in rectal swabs, the spread of the virus through fecal-oral route or contact is also possible(13). On February 2, 2020, a case of intrauterine infection was reported by Wuhan local media. The media reported that a confirmed positive pregnant woman gave birth to a positive newborn under strict level three protection(13). However, according to one study, amniotic fluid, cord blood, newborn's throat swab, and breast milk from six confirmed positive pregnant women were tested and reported negative, which suggests that there is currently no evidence for intrauterine infection(18).

Bats were initially considered to be the most likely natural reservoir of SARS-CoV-2, but differences between the bat-Coronavirus sampled and SARS-CoV-2 suggested that the humans were infected via an intermediate host ${ }^{(19)}$. Confirmed by the phylogenetic analysis, all SARS-CoV-2 sequences match very closely with Bat SARS-like sequences, with Bat-SLRatG13 being the closest match to the SARSCoV-2 genomic sequence(overall 97\%, 96\% for S1, and $100 \%$ identity for S2)(20). As the sequence of Receptor Binding Domain(RBD) of SARS-CoV-2 matches closely with the RBD sequences found in the Malayan Pangolins(M. Javanica) of Guangxi( $97 \%$ identical $)$, it is believed that these mammals might be the intermediate hosts $(20,21)$.

According to one study, on plastic surfaces, the viruses were viable for $72 \mathrm{~h}$, but the viral load decreased exponentially from $10^{3.7}$ to $10^{0.6}$ TCID $_{50}$. On stainless surfaces, the viral load decreased from $10^{3.6}$ to $10^{0.6}$ after 48h. On copper surfaces, no viable SARS-CoV and SARS-CoV- 2 were detected after $8 \mathrm{~h}$ and $4 \mathrm{~h}$ respectively. On cardboard, it took $24 \mathrm{~h}$ and $8 \mathrm{~h}$ to disappear completely for SARS-CoV and SARS-CoV-2 respectively. In aerosol-generating experiments, it took $3 \mathrm{~h}$ to become virus nonviable and the titers of SARS-CoV and SARSCoV-2 decreased from $10^{4.3}$ to $10^{3.5}$ and from $10^{3.5}$ to $10^{2.5}$ respectively(22).

Despite the low death ratio, SARS-CoV-2 caused more infections and deaths than SARS$\mathrm{CoV}$. It's because the infectivity of SARS-CoV-2 is far greater than SARS-CoV. There are some structural changes in Spike proteins of SARS$\mathrm{CoV}$ and SARS-CoV-2. Those are mainly found at the $\mathrm{S} 1 / \mathrm{S} 2$ cleavage site and in the RBD. Due to these structural changes, the affinity of SARSCoV-2 Spike protein towards the hACE2 has been increased $(5,23)$. This increased affinity might explain the increase in the infectivity of SARS-CoV-2 compared to SARS-CoV.

The common structure of the Spike protein the SARS Coronaviruses and differences between the Spike proteins of the SARS-CoV and SARS-CoV-2 are discussed in the following parts of the paper. 
Structure of the SARS

Coronaviruses and their

Spike Glycoproteins

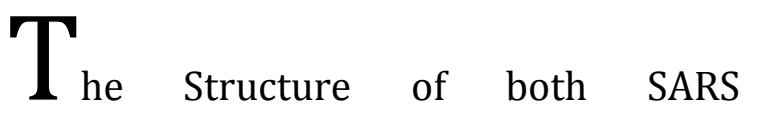

Coronaviruses is very identical. The genomic structure of both viruses is as follows: 5'-UTRreplicase-S(Spike protein)-E(Envelope protein)M(Membrane protein)-N(Nucleocapsid)-3'UTR Poly(A) tail $(3,24)$. M and E proteins are important for viral assembly and $N$ protein is necessary for RNA synthesis(13,24). The S protein(also known as Spike protein or Spike glycoprotein) is responsible for receptor binding and viral entry into the host cells $(2,24)$.

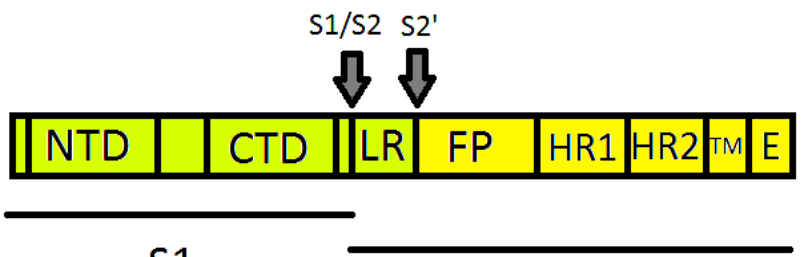

S1

S2

Figure 1. Regions of the Spike glycoprotein monomer. NTD : N TERMINAL DOMAIN CTD : C TERMINAL DOMAIN S1/S2: 1ST CLEAVAGE SITE LR : LINKER REGION FP : FUSION PEPTIDE

HR1 : HEPTAD REPEAT 1

HR2 : HEPTAD REPEAT 2

TM : TRANS-MEMBRANE MOTIF

E : ENDODOMAIN

The Coronavirus Spike glycoprotein is a class 1 viral fusion protein that mediates virus binding and membrane fusion. It is a trimer made of 3 identical monomers. As observed in other class 1 fusion proteins, Each monomer of Spike glycoprotein also contains two functional domains; S1 and S2, linked by a protease cleavage site called S1/S2 cleavage site(2). There is another cleavage site known as S2' cleavage site, which is located directly upward to Fusion peptide.

The S1 contains N-Terminal Domain (NTD) and C-Terminal Domain (CTD). The S2 contains four regions named Fusion Peptide, Heptad Repeats(HR1 \& HR2), Transmembrane Domain, and an intracellular Endodomain. The CTD of S1 contains a region named Receptor Binding Domain(RBD) which binds with its receptor, hACE2(Human AngiotensinConverting Enzyme 2). Inside the RBD, an amino acid motif called Receptor Binding Motif(RBM) makes most of the connections with hACE2(25,26).

The Fusion peptide is typically composed of 15-25-amino acids, generally hydrophobic (particularly enriched in Glycine $(G)$ and Alanine(A) residues), which becomes anchored within the target membrane. The Heptad regions are considered the key structures of the membrane fusion machinery, they are repetitive heptapeptides containing some hydrophobic amino acid residues. Once the Fusion peptide is inserted into the host cell membrane, Heptad regions start to change their structure and form a compact structure called 6HB, which eventually leads to the cell membrane fusion between the virus and the host cell. Transmembrane Domain is embedded into the viral membrane and the Endodomain is a part of the $S$ protein, which is present inside the virus $(25,26)$. Fusion peptide(further divided in FP1 and FP2 regions), HR1, and HR2 are together called the Fusion domain.

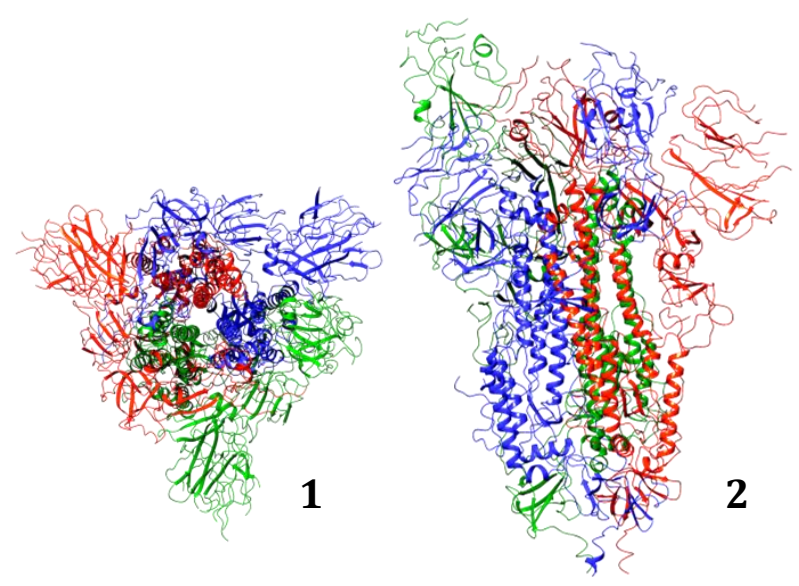

Figure 2. Cartoon representation of trimeric structure of the Spike glycoprotein of SARS-CoV-2.

1. TOP view

2. SIDE view

The SARS-CoV and SARS-CoV-2 both use the hACE2(Human Angiotensin Converting Enzyme 2) as a gateway to enter the human cells ${ }^{(5,27)}$. ACE2 is present on the cell membrane of the human cells. It is a part of the RAAS(Renin-Angiotensin-Aldosterone System). N-Terminal Peptidase Domain of ACE2(S19D615) connects with the RBM of S1. The NTerminal Peptidase Domain Of ACE2 has two lobes, forming the peptide substrate binding site between them. The extended RBM in the 
SARS-CoV-2 RBD contacts the bottom side of the small lobe of ACE2, with a concave outer surface in the RBM that accommodates the $\mathrm{N}$-Terminal helix of the ACE2(5).

\section{Structural comparison of The Spike protein of SARS Coronaviruses}

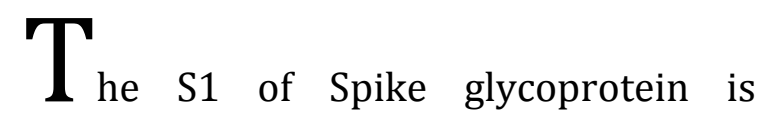
observed in two conformations; UP and DOWN. At least one of the three S1 domains of a fully functional Spike protein is required to be in UP conformation in order to bind with the hACE2. In SARS-CoV Spike protein, the angle between the $\mathrm{S} 1$ and the stem of the protein(S2 mainly) in DOWN conformation is found to be approximately $22.7^{\circ}$, and in UP conformation it is between $50^{\circ}$ and $70^{\circ}$. When the $\mathrm{S} 1$ is bound to the hACE2, the angle between S1 and S2 is observed in the range of $50^{\circ}$ to $111.6^{\circ}$. In approximately $19 \%$ of the hACE2-bound particles, an angle of $111.6^{\circ}$ is observed, which is not observed in hACE2-free particles ${ }^{(28)}$.

The Receptor Binding Domain(RBD) contains five anti-parallel $\beta$-sheets $(\beta 1-4 \quad \& \quad \beta 7)$ connected with short helices and loops that make the core. The extended insertion between

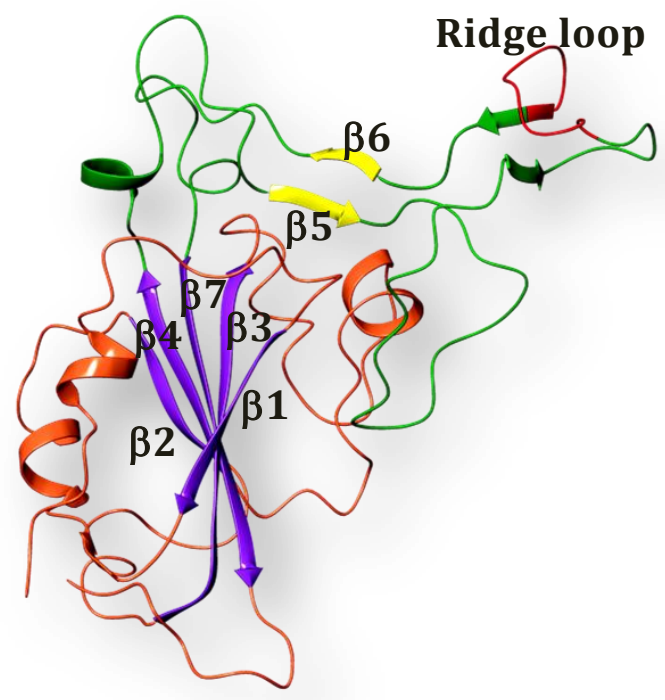

Figure 3. Cartoon representation of the structure of RBD of the SARS-CoV-2.

B1-4 \& 7: Purple

$\beta 5$ \& 6: Yellow

RBM: Green

Cys480-488(Ridge loop): Red $\beta 4 \& \beta 7$ is called Receptor Binding Motif(RBM). It is made of $\beta 5 \& \beta 6$ sheets and $\alpha 4 \& \alpha 5$ helices and loops. There are four disulfide bonds present in the structure of RBD, three of them are in the core which help to stabilize the $\beta$ sheets and the remaining one(Cys480-Cys488 and Cys467-Cys474 for SARS-CoV-2 and SARS$\mathrm{CoV}$, respectively) connects the loops in the distal end of the $\operatorname{RBM}(5,27,29)$.

The Methionine(82) and Lysine(353) are two important residues of hACE2. Mutation in one of these two amino acids renders the ACE2 less effective as seen in mouse-ACE2 that contains a Histidine at position 353. Mutation in both these two amino acids renders the ACE2 ineffective as seen in rat-ACE2 that contains a glycosylated Asparagine at position 82 and the Histidine residue at position $353^{(30)}$. The Methionine(82) residue has Van-der-Waals connections with the Phe(486) of SARS-CoV-2 and Leucine(472) of SARS-CoV. The Lysine(353) has Van-der-Waals connections with Gly(496)*, Asn(501), Gly(502)*, Tyr(505) of SARS-CoV-2 and with $\operatorname{Tyr}(481), \mathrm{Gly}(482)$, Tyr(484), Thr(487), Gly(488)*, $\operatorname{Tyr}(491)$ of SARS-CoV(with vdw cut-off set to $4.5 \mathrm{~A}^{\circ}$ and $\mathrm{H}$ bonds cut-off set to $3.5 \mathrm{~A}^{\circ}$; ${ }^{*}$ forms $\mathrm{H}$-bond) ${ }^{(31)}$.

With distance cut-off set to $4 \mathrm{~A}^{\circ}$, a total of 16 and 17 residues are found in contact with 20 residues of hACE2 for SARS-CoV and SARS-CoV2 , respectively. 17 residues out of those 20 residues of hACE2 are shared for both RBMs. 14 of those residues belonging to RBM are shared by both RBMs to interact with hACE2; 8 of them are exactly same residues, other 5 are having similar biochemical properties, and the remaining one is Tyrosine(484) and Glutamine(498) for SARS-CoV and SARS-CoV-2, respectively(Table 2$)^{(5)}$.

Among the six RBD positions with changed residues, four residues of SARS-CoV RBD are identified as essential residues for binding with hACE2. Tyr(442) of SARS-CoV is replaced with Leu(455) of SARS-CoV-2, they both interact in a similar way with Asp(30), Lys(31), His(34) of hACE2. Leu(472) of SARS$\mathrm{CoV}$ is replaced with Phe(486) of SARS-CoV-2, Phe(486) interacts with $G \ln (24)$, Leu(79), Met(82), Tyr(83) of hACE2, whereas Leu(472) has less contact with Leu(79) and Met(82) of hACE2. Asn(479) of SARS-CoV is replaced with 
$\mathrm{Gln}(493)$ of SARS-CoV-2, Gln(493) interacts with Lys(31), His(34) and Glu(35) of hACE2 and forms a hydrogen bond with Glu(35), whereas Asn(479) of SARS-CoV interacts only with His(34). Thr(487) of SARS-CoV is replaced with Asn(501) of SARS-CoV-2, they both form a hydrogen bond with Tyr(41) of hACE2(5).

There is a ridge loop present in the structure of the RBM of both Spike proteins. It is bound with a disulfide bond(Cys480-Cys488 and Cys467-Cys474 for SARS-CoV-2 and SARS$\mathrm{CoV}$, respectively). Excluding bond-forming Cysteines, the ridge loop of SARS-CoV is made of an amino acid chain containing six amino acids (468 TPPAFN $_{473}$ ) and the ridge loop of SARS$\mathrm{CoV}-2$ is made of an amino acid chain containing seven amino acids $\left(481 \text { NGVEGFN }_{487}\right)^{(32,33)}$. by Tyrosine(442) residue of Spike protein. As it is stabilized, it doesn't make any bond with the RBM of the Spike protein. But in SARS-CoV-2 RBM/hACE2 interface, Leucine(455) from SARS-CoV-2 RBM (corresponding to Tyrosine(442) from SARS-CoV RBM) has a less bulky side chain, providing less support for Lysine(31) from hACE2. As a result, the salt bridge between Lysine(31) and Glutamate(35) breaks apart and each of the residues forms a hydrogen bond with Glutamine(493) from SARS-CoV-2 RBM(34). Outside of the RBM, there is a unique hACE2-interacting residue Lys(417) in the Spike protein of SARS_CoV-2, which forms a salt bridge with Asp(30) of hACE2, this position is replaced by Valine in the Spike protein of SARS-CoV, which fails to create a bond(5).
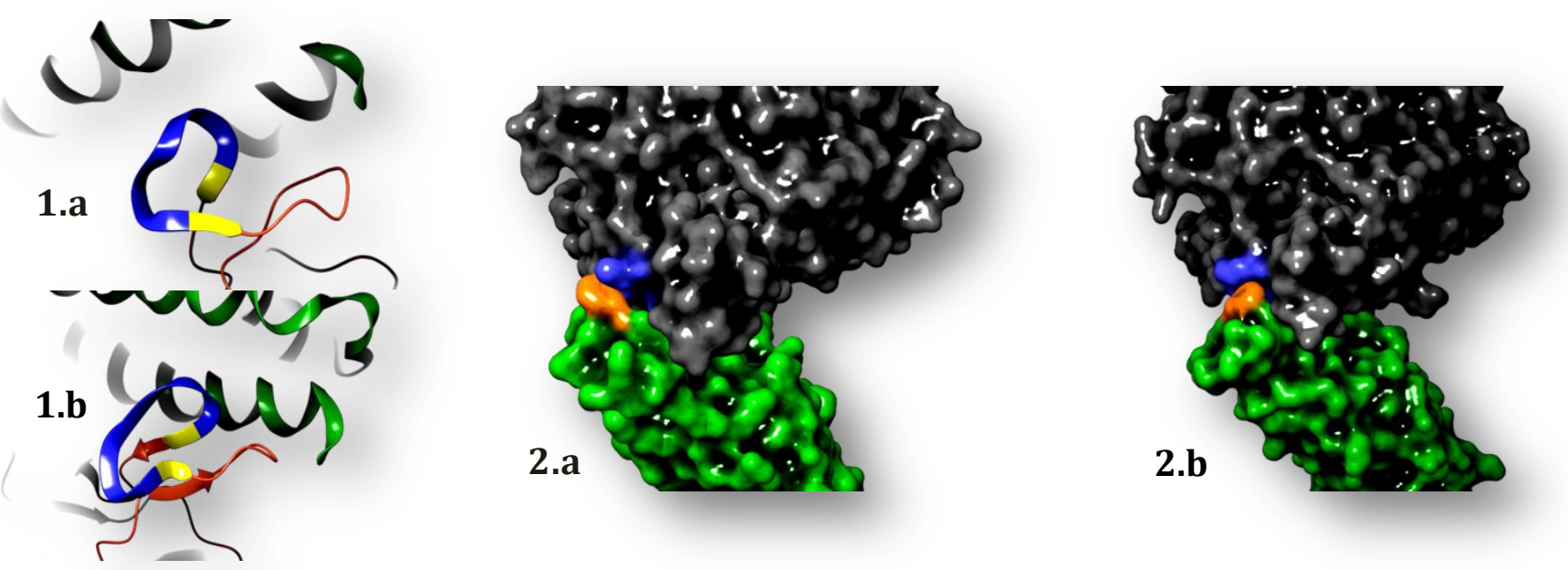

Figure 4.(1)Structure of the ridge loop of the SARS-Coronaviruses and (2)Relative position of Phenylalanine residue of $\mathrm{RBM}$ in comparison of Methionine(82), Leucine(79), and Tyrosine(83) residues from hACE2.

[4.1] YELLOW : CYSTEINE RESIDUES FORMING THE DISULFIDE BOND BLUE : VARIABLE REGION OF THE LOOP GREEN : PART OF THE hACE2

[a]: SARS-COV(1 - CYS474-CYS467)(2 - F)

[b]: SARS-COV-2(1 - CYS480-CYS488)(2 - F)

The Asparagine residue of the ridge loop(Asn(473) and Asn(487) of SARS-CoV and SARS-CoV-2, respectively) creates $\mathrm{H}$-bonds with the Glutamine(24) and Tyrosine(83) residues of the hACE2. Compared with the Phenylalanine(472) residue of the ridge loop belonging to SARS-CoV, Phenylalanine(486) from the SARS-CoV-2 ridge loop points to a different direction and inserts into a hydrophobic pocket made of Methionine(82), Leucine(79), and Tyrosine(83) residues of hACE2 (34).

A salt bridge between Lysine(31) and Glutamate(35) is found in the structure of hACE2. In case of the SARS-CoV, it is stabilized
[ 4.2] GREY : PORTION OF hACE2 GREEN : PORTION OF S1 RBD BLUE : POCKET MADE OF METHIONINE(82), LEUCINE(79), AND TYROSINE(83) RESIDUES

ORANGE : PHENYLALANINE RESIDUE

The S1/S2 cleavage site is present at $\mathrm{R}(667)-\mathrm{S}(668)$ and $\mathrm{R}(685)-\mathrm{S}(686)$ positions for SARS-CoV and SARS-CoV-2, respectively. The S1/S2 cleavage site of SARS-CoV-2 Spike protein contains an additional amino acid insertion ${ }_{681}$ PRRA $_{684}$ that is not found in the Spike protein of SARS-CoV(5,20,35,36). The S1/S2 cleavage site of the Spike protein of SARA-CoV forms a short loop that is positioned very close to the trimeric structure(20).

The priming of Spike protein at S1/S2site is relatively a straight forward process compared to the priming of Spike protein at S2'site. The S2'-site can only be cleaved after the binding of RBD with hACE2. A C-shaped amino- 
acid chain containing 10 amino-acid residues covers the S2'-site. This chain is clipped by the Linker region amino-acid chain of adjacent monomer. Disassociation of one S1/hACE2 complex from the Spike protein releases the clipping Linker region chain and induces conformational changes in the C-shaped aminoacid chain, exposing the S2'-site for protease cleavage.

At the S2' site, Arginine at position P1 as well as Serine at position $\mathrm{P}^{\prime}$ ' both are conserved in the case of SARS-CoV-2. The mutations Leucine to Serine and Threonine to Serine are observed at positions P3 and P6 in the S2' cleavage site of Spike protein of SARSCoV-2 in comparison to SARS-CoV. For SARS$\mathrm{CoV}$, The FP1 of Fusion peptide is a polypeptide chain containing 18 amino acids. The FP1 of

\section{Discussion}

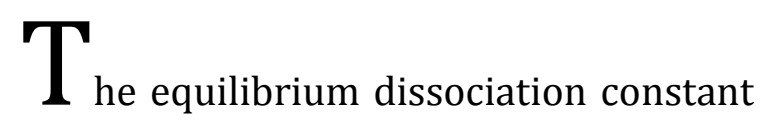

$\left(K_{\mathrm{D}}\right)$ of the hACE2/SARS-CoV-2 RBD complex is $4.7 \mathrm{nM}$, which is low in comparison to $K_{\mathrm{D}}$ of the hACE2/SARS-CoV RBD complex(31nM)(5). According to another study(23), the $K_{\mathrm{D}}$ of the hACE2/SARS-CoV-2 RBD complex was observed $14.7 \mathrm{nM}$ and the $K_{\mathrm{D}}$ of the hACE2/SARS-CoV RBD complex was observed $325 \mathrm{nM}$. In both studies, the $K_{\mathrm{D}}$ of SARS-CoV-2 RBD complex is low than the $K_{\mathrm{D}}$ of SARS-CoV RBD complex, which suggests that the stability of the SARS-CoV-2 $\mathrm{RBD} / \mathrm{hACE} 2$ complex is greater than the SARSCoV RBD/hACE2 complex.

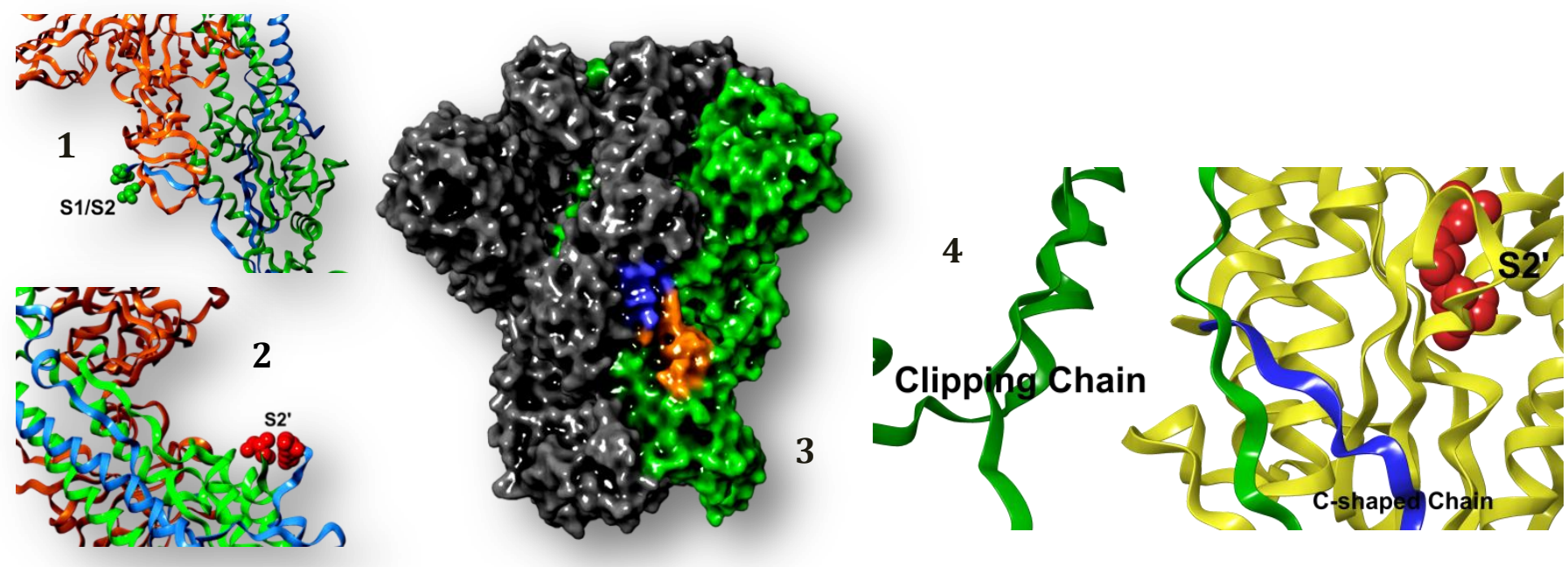

Figure 5. Structure of the SARS-CoV-2 S1/S2 and S2' cleavage sites.

1. S1/S2 CLEAVAGE SITE REPRESENTED AS GREEN ATOMS.

2. S2' CLEAVAGE SITE REPRESENTED AS RED ATOMS.

3. C-SHAPED LOOP(ORANGE) CLIPPED BY THE CLIPPING CHAIN OF OTHER MONOMER(BLUE).

4. CARTOON REPRESENTATION OF THE S2' CLEAVAGE SITE(RED ATOMS) MASKED BY THE C-SHAPED CHAIN(BLUE)(WHICH IS CLIPPED BY THE CLIPPING CHAIN OF OTHER MONOMER - GREEN).

both SARS viruses contains a highly conserved region of the "IEDLLF" amino acid sequence. These amino acids are very important for fusion. The highly conserved "LLF" motif corresponds to the beginning of a major antigenic determinant of SARS-CoV Spike protein, capable of eliciting the generation of neutralizing antibodies. The FP2(of SARS-CoV) is made of 21 amino acids and also has the ability to fuse with the membrane. The Fusion peptide is predicted to be in more compact conformation for SARS-CoV-2 than in SARS-CoV Spike protein $(20,37)$.
The overall protein sequence identity between the Spike proteins of SARS-CoV and SARS-CoV-2 is $76 \%$. The S1 is $64 \%$ conserved with the NTD having 51\% identity and RBD having 74\% identity. The identity of RBM is only $50 \%$. The S2 is well conserved(90\% identity) compared to S1. the Fusion peptides contain 93\% conserved sequence, Heptad repeat- 1 and Heptad repeat- 2 are having $88 \%$ and $100 \%$ identity, respectively. The Trans-membrane region and Endodomain are also having high conservation( $93 \%$ and $97 \%$ for $\mathrm{TM}$ and $\mathrm{E}$, respectively) ${ }^{(20) .}$ 
The increase in the angle between the S1 and the S2 suggests that binding of RBD with hACE2 leads to conformational changes in the structure of the Spike protein. Some pieces of evidence suggest the dissociation of the S1hACE2 complex. These Structural changes promote the priming of Spike protein at the S2' cleavage site.

Compared to SARS-CoV RBD having one H-bond(by Gly(488)) with The Lysine(353) of hACE2, The Lysine(353) residue of hACE2 is connected to SARS-CoV-2 RBD with two $\mathrm{H}$ bonds(with Gly(496) and Gly(502)). This additional hydrogen bond further stables the RBD/hACE2 complex. The insertion of Phenylalanine(486) from the SARS-CoV-2 ridge loop points into the hydrophobic pocket made of Methionine(82), Leucine(79), and Tyrosine(83) residues of hACE2 further stabilizes the complex(Figure 4.2).

The length of Asparagine-Glutamine(24 of hACE2) bond is $2.74 \mathrm{~A}^{\circ}$ and $2.69 \mathrm{~A}^{\circ}$ and the length of Asparagine-Tyrosine(83 of hACE2) bond is $3.00 \mathrm{~A}^{\circ}$ and $2.79 \mathrm{~A}^{\circ}$ for SARS-CoV and SARS-CoV-2, respectively. As mentioned earlier, $\operatorname{Thr}$ (487) of SARS-CoV is replaced with Asn(501) of SARS-CoV-2, they both form a hydrogen bond with Tyr(41) of hACE2. The average bond length of the bond between Thr(487) of SARS-CoV and Tyr(41) of hACE2 is $3.976 \mathrm{~A}^{\circ}$ and the average bond length of the bond between Asn(501) of SARS-CoV-2 and Tyr(41) of hACE2 is $3.723 \mathrm{~A}^{\circ}$. In both cases, the bond lengths for the SARS-CoV-2/hACE2 complex is shorter than the SARS-CoV/hACE2 complex, which suggests the strong bonding
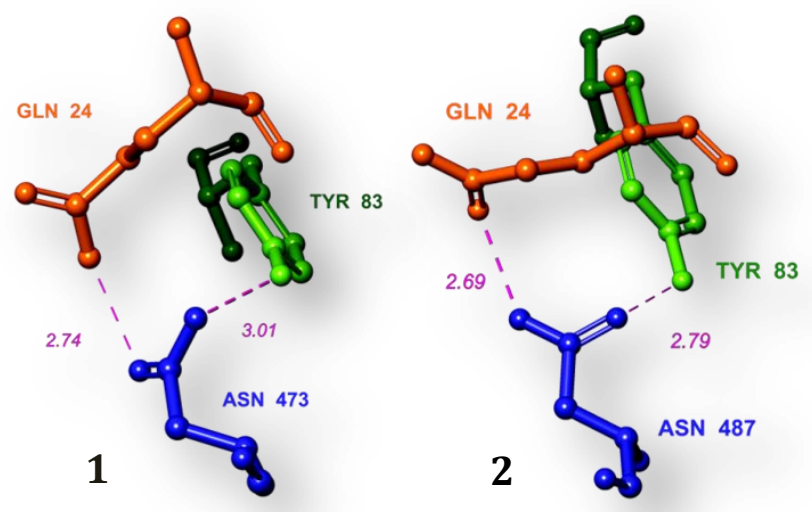

Figure 6. Asparagine-Glutamine \& AsparagineTyrosine bonds of hACE2/S-protein complex with their bond-lengths in $A^{\circ}$.

1: SARS-COV

2 : SARS-COV-2 between the SARS-CoV-2 and hACE2 compared to the SARS-CoV and hACE2.

The S1/S2 cleavage site and S2' cleavage site both can be cleaved by the TMPRSS proteases(particularly TMPRSS2 aka Serine protease 10). TMPRSS proteases are type-2 Transmembrane proteins. They are widely expressed in the respiratory tract and are involved in the activation of many respiratory viruses including both SARS-CoV and SARS-CoV2. They process the Spike protein on the cell surface during the entry of the virus inside the cell. The substrate specificity of TMPRSS proteases is not well defined, it is generally thought to be similar to that of Trypsin. Trypsin strongly prefers to cleave at Arginine(R) or Lysine $(\mathrm{K})$ residues, although almost all viral substrates get cleaved at Arginine(R)(26).
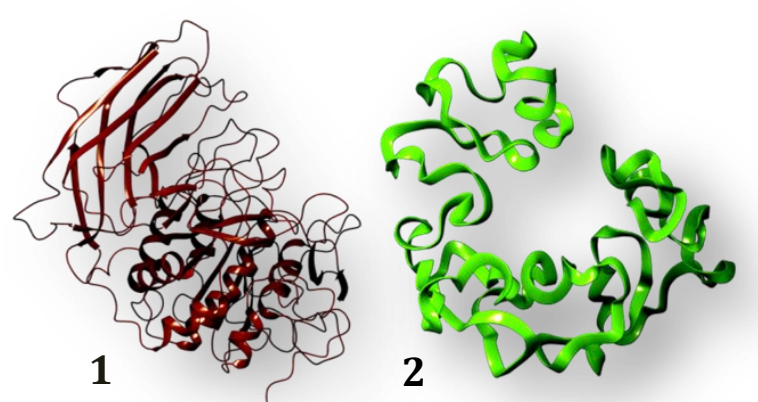

Figure 7. Structure of common proteolytic enzymes

\section{Structure of the Furin}

2.Predicted(using I-TASSER) structure of LDLreceptor class A domain of human TMPRSS2

Human TMPRSS2 is 492 amino acid long protein with three functional domains: an Nterminal LDL receptor class A domain(113-149) followed by SRCR(150-242) and finally a Cterminal Peptidase S1 domain spanning from 256 to 487 amino acid. It is first cleaved into two functional domains named Transmembrane protease serine 2 non-catalytic chain and catalytic chain(38)(39-41).

Due to the inserted Amino acid residues in the S1/S2 cleavage site of the SARS-CoV-2, it forms the extended loop that protrudes to the exterior of the trimeric structure. Because of that, the S1/S2 cleavage site of SARS-CoV-2 Spike protein is more exposed to enzymes compared to the SARS-CoV. Because of more exposure to the proteolytic enzymes, it becomes easier for the SARS-CoV-2 Spike protein to be primed at S1/S2 cleavage site compared to the SARS-CoV Spike protein(20). 
The inserted amino acid sequence ${ }_{681}$ PRRA $_{684}$ inside the S1/S2 cleavage site of the SARS-CoV-2 can be recognized by the proprotein-convertases(PCs). Furin is an important member of pro-protein-convertases(PCs). Furin is a serine protease, containing a critical Serine residue in its catalytic triad. Furin belongs to a subset of PCs that includes proproteinconvertase1(PC1), PC2, PC4, PC5, paired basic amino-acid cleaving enzyme4(PACE4) and PC7, which generally cleave at single or paired basic residues within the following motif,

$\mathrm{R} / \mathrm{K}-(\mathrm{X})$ 0, 2, 4, 6 - R/K ( X: any amino-acid), which means that any $\mathrm{X}$ amino-acid in either 2 , 4 , or 6 numbers between two $\mathrm{R} / \mathrm{K}$ residues( ${ }^{(26)}$.

The minimal substrate recognition motif for Furin is $\mathrm{R}-\mathrm{X}-\mathrm{X}-\mathrm{R}$, with the R-X-R/K-R motif being highly favorable. The fairly strict requirement for basic Arginine(R) at $\mathrm{P} 1$ and $\mathrm{P} 4$ positions(as well as Lysine $(\mathrm{K})$ at $\mathrm{P} 2$ is due to Furin's binding pocket containing complementary charged residues. Serine(S) residues in the vicinity of the cleavage site appear to be highly favored by Furin. Selfactivation of Furin via protonation of a regulatory Histidine residue(His69) requires a slightly acidic environment. Furin is highly expressed in lungs ${ }^{(35)}$, it is produced in the ER and traffics along the secretory pathway. It cleaves the Spike protein of SARS-CoV-2 during viral assembly and delivery to the cell surface ${ }^{(20,26) \text {. }}$
No Furin cleavage site was found during the analysis of SARS-CoV Spike glycoprotein. Due to the absence of a Furin cleavage site, it is compulsory for the SARS-CoV Spike protein to be cleaved at both sites while entering the target host cell. As SARS-CoV-2 Spike protein contains a Furin cleavage site, it has the choice to be cleaved at the S1/S2-site during the production of viral proteins inside the infected host cell. Therefore on the target host cell surface, it needs to be primed at only one site(S2') while entering the host cell. This might lead to increased infectivity of the virus as it makes the entry of the virus into the host cell more easy and flexible.

The mutations Leucine to Serine and Threonine to Serine, observed at positions P3 and P6 in the S2' cleavage site of Spike protein of SARS-CoV-2, are predicted not to alter the structure of S2' cleavage site. As the Fusion peptides of both viruses contain conserved functional motifs, these regions are also not believed to have differences in their functionality $(20,37)$.

Therefore, it can be said that the increase in affinity of SARS-CoV-2 towards the hACE2 is mainly because of structural changes in the RBD region and the region of the S1/S2 cleavage site. The increased infectivity of the SARS-CoV-2 virus might be directly related to the increased stability of SARS-CoV-2 RBD/hACE2 complex.

Molecular images are created using the Maestro and the PyMOL(42,43). 
Table 1. Classification of Amino Acids With Their One-letter Abbreviations

\begin{tabular}{|c|c|c|}
\hline Amino Acid & $\begin{array}{l}\text { Short } \\
\text { Name }\end{array}$ & $\begin{array}{c}\text { One Letter } \\
\text { Abbreviation }\end{array}$ \\
\hline $\begin{array}{l}\text { Amino Acids With Positively } \\
\text { Charged Side Chains }\end{array}$ & - & - \\
\hline Arginine & $\operatorname{Arg}$ & $\mathrm{R}$ \\
\hline Histidine & His & $\mathrm{H}$ \\
\hline Lysine & Lys & K \\
\hline $\begin{array}{c}\text { Amino Acids with Negatively } \\
\text { Charged Side Chains }\end{array}$ & - & - \\
\hline Aspartic Acid & Asp & D \\
\hline Glutamic Acid & Glu & $E$ \\
\hline $\begin{array}{l}\text { Amino Acids With Polar } \\
\text { Uncharged Side Chains }\end{array}$ & - & - \\
\hline Serine & Ser & $S$ \\
\hline Threonine & Thr & $\mathrm{T}$ \\
\hline Asparagine & Asn & $\mathrm{N}$ \\
\hline Glutamine & Gln & $Q$ \\
\hline Special Cases & - & - \\
\hline Cysteine & Cys & C \\
\hline Glycine & Gly & G \\
\hline Proline & Pro & $P$ \\
\hline $\begin{array}{l}\text { Amino Acids With Hydrophobic } \\
\text { Side Chains }\end{array}$ & - & - \\
\hline Alanine & Ala & $A$ \\
\hline Valine & Val & V \\
\hline Isoleucine & Ile & 1 \\
\hline Leucine & Leu & $\mathrm{L}$ \\
\hline Methionine & Met & M \\
\hline Phenylalanine & Phe & $\mathrm{F}$ \\
\hline Tyrosine & Tyr & $Y$ \\
\hline Tryptophan & $\operatorname{Trp}$ & w \\
\hline
\end{tabular}


Table 2. Amino acid positions shared by both RBMs to interact with the ACE2

\begin{tabular}{|c|c|}
\hline SARS-CoV & SARS-CoV-2 \\
\hline \multicolumn{2}{|c|}{ POSITIONS WITH IDENTICAL AMINO ACIDS } \\
\hline Tyr(436) & Tyr(449) \\
\hline $\operatorname{Tyr}(440)$ & Tyr(453) \\
\hline Asn(473) & Asn(487) \\
\hline $\operatorname{Tyr}(476)$ & Tyr(489) \\
\hline Gly(482) & Gly(496) \\
\hline $\operatorname{Thr}(486)$ & $\operatorname{Thr}(500)$ \\
\hline Gly(488) & Gly(502) \\
\hline $\operatorname{Tyr}(491)$ & $\operatorname{Tyr}(505)$ \\
\hline \multicolumn{2}{|c|}{ POSITIONS CONTAINING AMINO ACIDS WITH SIMILAR BIOCHEMICAL PROPERTIES } \\
\hline $\operatorname{Tyr}(442)$ & Leu(455) \\
\hline Leu(443) & Phe(456) \\
\hline Leu(472) & Phe(486) \\
\hline Asn(479) & $G \ln (493)$ \\
\hline $\operatorname{Thr}(487)$ & Asn(501) \\
\hline \multicolumn{2}{|c|}{ POSITIONS CONTAINING BIOCHEMICALLY DIFFERENT AMINO ACIDS } \\
\hline Tyr(484) & $G \ln (498)$ \\
\hline
\end{tabular}

- $\quad$ Analyzed with the distance cut-off set to $4 A^{\circ}(5)$. 
Table 3. Comparison of hACE2 Binding to SARS-CoV-2-RBD and SARS-RBD

\begin{tabular}{|c|c|c|}
\hline hACE2 & SARS-CoV-2-RBD & SARS-RBD \\
\hline S19 (7/1) & A475 (3,ㅅ), G476 (4) & P462 (1) \\
\hline Q24 (24/6) & $\begin{array}{c}\text { A475 (4), G476 (5), } \\
\text { N487 }(15, \underline{1})\end{array}$ & N473 $(6, \underline{1})$ \\
\hline T27 (15/8) & $\begin{array}{l}\text { F456 (5), Y473 (1), } \\
\text { L443 (3), Y475 (5) } \\
\text { A475 (2), Y489 (7) }\end{array}$ & L443 (3), Y475 (5) \\
\hline F28 (7/7) & Y489 (7) & Y475 (7) \\
\hline D30 (10/2) & $\begin{array}{l}\text { K417 (4, 1), L455 (2), } \\
\text { Y442 (2) } \\
\text { F456 (4) }\end{array}$ & Y442 (2) \\
\hline K31 (19/12) & $\begin{array}{c}\text { L455 (2), F456 (5), } \\
\text { Y442 (6), Y475 (6) } \\
\text { E484 (1), Y489 (6), F490 (2), Q493 (3) }\end{array}$ & Y442 (6), Y475 (6) \\
\hline H34 (20/10) & $\begin{array}{c}\mathrm{Y} 453(5, \underline{1}), \mathrm{L} 455(9) \\
\mathrm{Y} 440(5,1) \\
\mathrm{Q} 493(6)\end{array}$ & $\begin{array}{c}\mathrm{Y} 440(5, \underline{1}) \\
\text { Q493 (6) } \\
\text { Y442 (1), N479 (4) }\end{array}$ \\
\hline E35 $(8 / 0)$ & Q493 (8) & - \\
\hline $\operatorname{E3} 7(7 / 4)$ & Y505 (7) & Y491 (4) \\
\hline D38 (15/11) & $\begin{array}{c}\text { Y449 (9, 1), G496 (5), } \\
\text { Y436 (9, 2), } \\
\text { Q498 (1) }\end{array}$ & $\begin{array}{c}\text { Y436 }(9,2) \\
\text { Q498 (1) } \\
\text { G482 (1), Y484 (1) }\end{array}$ \\
\hline Y41 (23/25) & $\begin{array}{c}\text { Q498 (8), T500 (7, } 1), \\
\text { N501 }(8, \underline{1})\end{array}$ & $\begin{array}{c}\text { Y484 (9), T486 }(8,1) \\
\text { T487 }(8)\end{array}$ \\
\hline Q42 (16/9) & $\begin{array}{c}\text { G446 (4, } \underline{1}), \text { Y Y449 }(4, \underline{1}) \\
\text { Q498 }(8, \underline{3})\end{array}$ & Y436 (5, 1), Y484 (4) \\
\hline L45 (4/3) & Q498 (3), T500 (1) & Y484 (2), T486 (1) \\
\hline $\mathrm{L} 79(2 / 2)$ & F486 (2) & L472 (2) \\
\hline M82 (9/4) & F486 (9) & L472 (4) \\
\hline Y83 (20/10) & $\begin{array}{c}\text { F486 (11), N487 }(8, \underline{1}), \\
\text { Y489 (1) }\end{array}$ & N473 $(8, \underline{2})$, Y475 (2) \\
\hline $\mathrm{Q} 325(0 / 4)$ & - & $\mathrm{R} 426(2), \mathrm{I} 489(2)$ \\
\hline $\operatorname{E329}(0 / 6)$ & - & $\mathrm{R} 426(6, \underline{1})$ \\
\hline N330 (8/11) & T500 (8) & $\mathrm{T} 486(11, \underline{1})$ \\
\hline K353 (50/48) & $\begin{array}{l}\text { G496 }(7, \underline{1}), \text { N501 (11), } \\
\text { G502 }(4, \underline{1}), \text { Y505 }(28)\end{array}$ & $\begin{array}{l}\text { Y481 (1), G482 (3), } \\
\text { Y484 (2), T487 (11), } \\
\text { G488 }(6, \underline{1}), \text { Y491 (25) }\end{array}$ \\
\hline G354 $(11 / 10)$ & Y502 (7), Y505 (4) & G488 (7), Y491 (3) \\
\hline D355 (9/15) & T500 (8, 1), G502 (1) & T486 (8), T487 (3), G488 (4) \\
\hline R357 (3/4) & $\mathrm{T} 500(3)$ & $\mathrm{T} 486(4)$ \\
\hline R393 (1/1) & Y505 (1) & Y491 (1) \\
\hline Total & $288(16)$ & $213(11)$ \\
\hline
\end{tabular}

- The numbers in parentheses of hACE2 residues represent the number of vdw contacts of the indicated residue with SARS-CoV-2-RBD and SARS-RBD, respectively.

- The numbers in parentheses of either S protein residues represent the numbers of vdw contacts the indicated residues conferred. The numbers with underline suggest a number of potential $\mathrm{H}$-bonds between the pairs of residues. vdw contact was analyzed at a cut-off of $4.5 \mathrm{~A}^{\circ}$ and H-bonds at a cut-off of $3.5 \mathrm{~A}^{\circ}(31)$. 


\section{References And Citations}

1. Hudson CB, Beaudette FR. Infection of the cloaca with the virus of infectious bronchitis. Vol. 76, Science. American Association for the Advancement of Science; 1932. p. 34.

2. Kasmi Y, Khataby K, Souiri A, Ennaji MM. Coronaviridae: 100,000 years of emergence and reemergence. Emerg Reemerging Viral Pathog Vol 1 Fundam Basic Virol Asp Human, Anim Plant Pathog. 2019;127-49.

3. Ma C, Su S, Wang J, Wei L, Du L, Jiang S. From SARS-CoV to SARS-CoV-2: safety and broad-spectrum are important for coronavirus vaccine development. Microbes Infect [Internet]. 2020;(November 2002). Available from: https://doi.org/10.1016/j.micinf.2020.05 .004

4. Benvenuto D, Giovanetti M, Ciccozzi A, Spoto S, Angeletti S, Ciccozzi M. The 2019new coronavirus epidemic: Evidence for virus evolution. J Med Virol. 2020;92(4):455-9.

5. Lan J, Ge J, Yu J, Shan S, Zhou H, Fan S, et al. Structure of the SARS-CoV-2 spike receptor-binding domain bound to the ACE2 receptor. Nature. 2020;

6. Vijgen L, Keyaerts E, Lemey P, Maes P, Van Reeth $K$, Nauwynck $H$, et al. Evolutionary History of the Closely Related Group 2 Coronaviruses: Porcine Hemagglutinating Encephalomyelitis Virus, Bovine Coronavirus, and Human Coronavirus OC43. J Virol. 2006;80(14):7270-4.

7. Coronavirus - Wikipedia [Internet]. [cited 2020 May 18]. Available from: https://en.wikipedia.org/wiki/Coronavir us

8. Su S, Wong G, Shi W, Liu J, Lai ACK, Zhou J, et al. Epidemiology, Genetic
Recombination, and Pathogenesis of Coronaviruses. Trends Microbiol [Internet]. 2016;24(6):490-502. Available from: http://dx.doi.org/10.1016/j.tim.2016.03. 003

9. Isaacs D, Flowers D, Clarke JR, Valman HB, MacNaughton MR. Epidemiology of coronavirus respiratory infections. Arch Dis Child. 1983;58(7):500-3.

10. Zhu N, Zhang D, Wang W, Li X, Yang B, Song J, et al. A novel coronavirus from patients with pneumonia in China, 2019. N Engl J Med. 2020;382(8):727-33.

11. Greenberg SB. Update on Human Rhinovirus and Coronavirus Infections. Semin Respir Crit Care Med. 2016;37(4):555-71.

12. Kaul D. An overview of coronaviruses including the SARS-2 coronavirus Molecular biology, epidemiology and clinical implications. Curr Med Res Pract [Internet]. 2020;10(2):54-64. Available from:

https://doi.org/10.1016/j.cmrp.2020.04. 001

13. Yang $Y$, Peng F, Wang R, Guan K, Jiang T, $\mathrm{Xu} \mathrm{G}$, et al. The deadly coronaviruses: The 2003 SARS pandemic and the 2020 novel coronavirus epidemic in China. J Autoimmun [Internet]. 2020;109(February):102434. Available from:

https://doi.org/10.1016/j.jaut.2020.1024 34

14. WHO | Summary table of SARS cases by country, 1 November 2002 - 7 August 2003. WHO. 2015;

15. Bartlett JG. Evaluation of control measures implemented in the severe acute respiratory syndrome outbreak in Beijing, 2003. Infect Dis Clin Pract. 2004;12(3):216-7.

16. Tan W, Zhao X, Ma X, Wang W, Niu P, Xu 
W, et al. Notes from the Field A Novel Coronavirus Genome Identified in a Cluster of Pneumonia Cases - Wuhan , China 2019 - 2020. China CDC Wkly. 2020;2(4):61-2.

17. COVID-19 Corona Tracker [Internet]. [cited 2020 May 18]. Available from: https://www.coronatracker.com/

18. Chen H, Guo J, Wang C, Luo F, Yu X, Zhang $\mathrm{W}$, et al. Clinical characteristics and intrauterine vertical transmission potential of COVID-19 infection in nine pregnant women: a retrospective review of medical records. Lancet [Internet]. 2020;395(10226):809-15. Available from: http://dx.doi.org/10.1016/S01406736(20)30360-3

19. Lu R, Zhao X, Li J, Niu P, Yang B, Wu H, et al. Genomic characterisation and epidemiology of 2019 novel coronavirus: implications for virus origins and receptor binding. Lancet. 2020 Feb 22;395(10224):565-74.

20. Jaimes JA, André NM, Chappie JS, Millet JK, Whittaker GR. Phylogenetic Analysis and Structural Modeling of SARS-CoV-2 Spike Protein Reveals an Evolutionary Distinct and Proteolytically Sensitive Activation Loop. J Mol Biol [Internet]. 2020;432(10):3309-25. Available from: https://doi.org/10.1016/j.jmb.2020.04.0 09

21. Lam TTY, Shum MHH, Zhu HC, Tong YG, $\mathrm{Ni} \mathrm{XB}$, Liao YS, et al. Identifying SARSCoV-2 related coronaviruses in Malayan pangolins. Nature. 2020;

22. Dorenmalen N, Bushmaker T MD. Aerosol and Surface Stability of SARS-CoV-2 as Compared with SARS-CoV-1. N Engl J Med. 2020;0-3.

23. Wrapp D, Wang N, Corbett KS, Goldsmith JA, Hsieh CL, Abiona 0, et al. Cryo-EM structure of the 2019-nCoV spike in the prefusion conformation. Science (80- ). 2020;367(6483):1260-3.
24. A. R. F, Perlman S. Coronaviruses: Methods and protocols. Coronaviruses Methods Protoc. 2015;1282(1):1-282.

25. Belouzard S, Millet JK, Licitra BN, Whittaker GR. Mechanisms of coronavirus cell entry mediated by the viral spike protein. Viruses. 2012;4(6):1011-33.

26. Lai AL, Millet JK, Daniel S, Freed JH, Whittaker GR. Host cell proteases: Critical determinants of coronavirus tropism and pathogenesis. Virus Res. 2015;202:12034.

27. Li F, Li W, Farzan M, Harrison SC. Structural biology: Structure of SARS coronavirus spike receptor-binding domain complexed with receptor. Science (80- ). 2005;309(5742):1864-8.

28. Song W, Gui M, Wang X, Xiang Y. Cryo-EM structure of the SARS coronavirus spike glycoprotein in complex with its host cell receptor ACE2. PLoS Pathog. 2018;14(8):1-19.

29. Spiga O, Bernini A, Ciutti A, Chiellini S, Menciassi N, Finetti $F$, et al. Molecular modelling of S1 and S2 subunits of SARS coronavirus spike glycoprotein. Biochem Biophys Res Commun. 2003;310(1):7883.

30. Li W, Greenough TC, Moore MJ, Vasilieva N, Somasundaran M, Sullivan JL, et al. Efficient Replication of Severe Acute Respiratory Syndrome Coronavirus in Mouse Cells Is Limited by Murine Angiotensin-Converting Enzyme 2. J Virol. 2004;78(20):11429-33.

31. Wang Q, Zhang Y, Wu L, Niu S, Song C, Zhang Z, et al. Structural and Functional Basis of SARS-CoV-2 Entry by Using Human ACE2. Cell. 2020;1-11.

32. RCSB PDB - 6MOJ: Dimeric DARPin A_angle_R5 complex with EpoR [Internet]. [cited 2020 May 18]. Available from:

https://www.rcsb.org/structure/6MOJ 
33. RCSB PDB - 3SCI: Crystal structure of spike protein receptor-binding domain from a predicted SARS coronavirus human strain complexed with human receptor ACE2 [Internet]. [cited 2020 May 18]. Available from: https://www.rcsb.org/structure/3SCI

34. Shang J, Ye G, Shi K, Wan Y, Luo C, Aihara $\mathrm{H}$, et al. Structural basis of receptor recognition by SARS-CoV-2. Nature [Internet]. 2020;1-8. Available from: http://dx.doi.org/10.1038/s41586-0202179-y

35. Coutard B, Valle C, de Lamballerie X, Canard B, Seidah NG, Decroly E. The spike glycoprotein of the new coronavirus 2019-nCoV contains a furin-like cleavage site absent in $\mathrm{CoV}$ of the same clade. Antiviral Res [Internet]. 2020;176(February):104742. Available from:

https://doi.org/10.1016/j.antiviral.2020. 104742

36. Markus Hoffmann HK-WSP. A multibasic cleavage site in the spike protein of SARSCoV-2 is essential for infection of human lung cells. Cell Press. 2020;1-6.

37. Millet JK, Whittaker GR. Physiological and molecular triggers for SARS-CoV membrane fusion and entry into host cells. Virology [Internet]. 2018;517(October 2017):3-8. Available from:

https://doi.org/10.1016/j.virol.2017.12.0 15

38. TMPRSS2 - Transmembrane protease serine 2 precursor - Homo sapiens (Human) - TMPRSS2 gene \& protein [Internet]. [cited 2020 May 14]. Available from:

https://www.uniprot.org/uniprot/01539 3

39. Yang J, Yan R, Roy A, Xu D, Poisson J, Zhang Y. The I-TASSER suite: Protein structure and function prediction. Nat Methods [Internet]. 2014;12(1):7-8.
Available from: http://dx.doi.org/10.1038/nmeth.3213

40. Zhang Y. I-TASSER server for protein 3D structure prediction. BMC Bioinformatics. 2008;9:1-8.

41. Roy A, Kucukural A, Zhang Y. I-TASSER: A unified platform for automated protein structure and function prediction. Nat Protoc. 2010;5(4):725-38.

42. Schrödinger L. The PyMOL Molecular Graphics System, Version 2.3.5 [Internet]. [cited 2020 May 18]. Available from: https://pymol.org/2/support.html?\#citin g

43. Schrödinger LNYN 2020. Schrödinger Release 2020-1: Maestro [Internet]. [cited 2020 May 18]. Available from: https://www.schrodinger.com/citations\# Maestro 